\title{
THE GEOMETRY OF WEAK RADON-NIKODYM SETS IN DUAL BANACH SPACES ${ }^{1}$
}

\author{
LAWRENCE H. RIDDLE
}

\begin{abstract}
Geometric characterizations in terms of trees, extreme points and dentability are presented for weak*-compact absolutely convex sets that have the RadonNikodym property for the Pettis integral.
\end{abstract}

I. Introduction. One useful method for investigating a property of Banach spaces is to localize the property by defining and studying it for an individual subset. Thus one encounters, for example, Dunford-Pettis sets, Radon-Nikodym sets, and recently Stegall's GSP sets, which are in some sense a localization of the Asplund property. In this paper we shall present several geometric characterizations of weak*-compact absolutely convex sets that have the Radon-Nikodym property for the Pettis integral. For operator theorists, such sets are characterized by the property that an operator on $L_{1}(\mu)$ mapping the unit ball of $L_{1}(\mu)$ into the set must be a DunfordPettis operator, i.e., must take weakly convergent sequences into norm convergent sequences. The characterizations presented here involve a new type of tree structure, a condition on the extreme points, and a dentability criterion due to Elias and Paulette Saab [11], and are based on a factorization lemma in Riddle, Saab, and Uhl [8].

Let us first fix some terminology. Throughout this paper $X$ and $Y$ will denote real Banach spaces. Let $(\Omega, \Sigma, \mu)$ be a finite measure space. A function $f(\cdot)$ from $\Omega$ into the Banach space $X$ is said to be Pettis integrable if the scalar function $x^{*} f(\cdot)$ is integrable for each $x^{*}$ in the dual space $X^{*}$ and if for each measurable set $E$ in $\Sigma$ there is an element $x_{E}$ of $X$ that satisfies

$$
x^{*}\left(x_{E}\right)=\int_{E} x^{*} f d \mu
$$

for every $x^{*}$ in $X^{*}$. In this case we write $x_{E}=$ Pettis- $\int_{E} f d \mu$.

A subset $K$ of $X$ is called a weak Radon-Nikodym set if for any finite measure space $(\Omega, \Sigma, \mu)$ and any vector measure $F: \Sigma \rightarrow X$ for which $F(E) \in \mu(E) K$ for every $E$ in $\Sigma$, there exists a Pettis integrable function $f: \Omega \rightarrow K$ such that $F(E)=$ Pettis- $\int_{E} f d \mu$ for every $E$ in $\Sigma$. A Banach space $X$ is said to have the weak Radon-Nikodym property if its closed unit ball, $B_{X}$, is a weak Radon-Nikodym set.

Received by the editors November 15, 1981.

1980 Mathematics Subject Classification. Primary 46G10, 46B20.

'This material constitutes a portion of the author's Ph.D. thesis from the University of Illinois under the direction of Professor J. J. Uhl, Jr. 
Recall that a set is weakly precompact if every sequence in the set has a weakly Cauchy subsequence. In his fundamental paper [10], Rosenthal proved that a bounded subset of a real Banach space $X$ is weakly precompact if and only if it contains no copy of the $l_{1}$-basis; that is, it is impossible to find a sequence $\left(x_{n}\right)$ in $X$ and a positive $\delta$ such that

$$
\left\|\sum a_{k} x_{k}\right\| \geqslant \delta \sum\left|a_{k}\right|
$$

for all finitely nonzero sequences $\left(a_{k}\right)$ of reals.

The cornerstone for our results will be the following theorem proved in Riddle, Saab, and Uhl [8].

THEOREM 1. Each of the following statements about an operator $T: X \rightarrow Y$ implies all the others:

(a) The set $T\left(B_{X}\right)$ is weakly precompact.

(b) The operator $T$ factors through a Banach space that contains no copy of $l_{1}$.

(c) The set $T^{*}\left(B_{Y^{*}}\right)$ is a weak Radon-Nikodym set.

(d) For any finite measure space $(\Omega, \Sigma, \mu)$ and any operator $S: L_{1}(\mu) \rightarrow X^{*}$ that maps the unit ball of $L_{1}(\mu)$ into $T^{*}\left(B_{Y^{*}}\right)$, the operator $S$ is a Dunford-Pettis operator.

Any weak*-compact absolutely convex set $K$ in the dual $X^{*}$ of a Banach space $X$ can be written in the form $K=T^{*}\left(B_{Y^{*}}\right)$. To see this, just take $Y$ to be the space $C(K)$ of continuous functions on $K$ and let $T: X \rightarrow Y$ be the evaluation operator defined by $T x\left(x^{*}\right)=x^{*}(x)$. This observation will allow us to use Theorem 1 to study arbitrary weak*-compact absolutely convex sets.

II. Rademacher trees. A sequence $\left(x_{n}\right)$ in a Banach space $X$ is called a tree if $x_{n}=\left(x_{2 n}+x_{2 n+1}\right) / 2$ for all $n=1,2, \ldots$ A tree is called a $\delta$-Rademacher tree [9] if there exists a $\delta>0$ such that

$$
\left\|x_{1}\right\| \geqslant \delta, \quad\left\|x_{2}-x_{3}\right\| \geqslant 2 \delta, \quad\left\|x_{4}-x_{5}+x_{6}-x_{7}\right\| \geqslant 4 \delta,
$$

and, in general,

$$
\left\|\sum_{n=2^{k}}^{2^{k+1}-1}(-1)^{n} x_{n}\right\| \geqslant 2^{k} \delta
$$

for all $k=0,1,2, \ldots$ Notice that each alternating sum is taken over an entire "row" of the tree.

Two of the best known trees are both Rademacher trees. Setting $x_{1}=\chi_{[0,1]}$, $x_{2}=2 \chi_{[0,1 / 2]}, x_{3}=2 \chi_{[1 / 2,1]}, x_{4}=4 \chi_{[0,1 / 4]}, x_{5}=4 \chi_{[1 / 4,1 / 2]}$, etc., produces a 1 Rademacher tree in $L_{1}[0,1]$. Also, letting $x_{1}=(1,0,0, \ldots), x_{2}=(1,1,0,0, \ldots)$, $x_{3}=(1,-1,0,0, \ldots), \quad x_{4}=(1,1,1,0, \ldots), x_{5}=(1,1,-1,0, \ldots), x_{6}=$ $(1,-1,1,0, \ldots), x_{7}=(1,-1,-1,0, \ldots)$, etc., yields a tree in the sequence space $c_{0}$ which is easily seen to be a 1-Rademacher tree.

THEOREM 2. For an operator $T: X \rightarrow Y$, the set $T^{*}\left(B_{Y^{*}}\right)$ is a weak Radon-Nikodym set if and only if it does not contain a Rademacher tree.

Consequently, a weak*-compact absolutely convex subset of $X^{*}$ is a weak Radon$N i_{1}$. Nom set if and only if it does not contain a Rademacher tree. 
Proof. Suppose $K=T^{*}\left(B_{Y^{*}}\right)$ contains a $\delta$-Rademacher tree $\left(x_{n}^{*}\right)$. Define the usual martingale associated with a tree (cf. [2, V.1.7]), i.e.,

$$
f_{1}=x_{1}^{*} \chi_{[0,1]}, \quad f_{2}=x_{2}^{*} \chi_{[0,1 / 2]}+x_{3}^{*} \chi_{[1 / 2,1]}
$$

etc. Define an operator $S: L_{1}[0,1] \rightarrow X^{*}$ by $S(g)=\lim _{n} \int g f_{n} d \lambda(\lambda$ is Lebesgue measure on $[0,1])$ and observe that if $r_{n}$ is the $n$th Rademacher function, then

$$
\left\|S\left(r_{n}\right)\right\|=\left\|\int r_{n} f_{n} d \lambda\right\| \geqslant \delta .
$$

Since the sequence $\left(r_{n}\right)$ is weakly null in $L_{1}[0,1]$, we see that the operator $S$ is not Dunford-Pettis. However, the set $K$ does contain $S\left(B_{L_{1}[0,1]}\right)$ since it contains the range of $f_{n}$ for each $n$. Invoke Theorem 1 to conclude that $K=T^{*}\left(B_{Y^{*}}\right)$ is not a weak Radon-Nikodym set.

Conversely, suppose $K=T^{*}\left(B_{Y^{*}}\right)$ is not a weak Radon-Nikodym set. Invoke Theorem 1 and Rosenthal's fundamental result [10] on weakly precompact sets to find a sequence $\left(T x_{n}\right)$ in $T\left(B_{X}\right)$ that is equivalent to the usual $l_{1}$-basis $\left(e_{n}\right)$. Let $Y_{0}$ be the closed subspace determined by the sequence $\left(T x_{n}\right)$. Then $S\left(T x_{n}\right)=e_{n}$ defines an isomorphism from $Y_{0}$ onto $l_{1}$. Let $\left(e_{n}^{*}\right)$ be the $c_{0}$-tree described earlier, but now considered as a tree in the sequence space $l_{\infty}$. Let $y_{n}^{*}=\|S\|^{-1} S^{*}\left(e_{n}^{*}\right)$. Then $\left(y_{n}^{*}\right)$ is a tree in $B_{Y_{0}^{*}}$. By the lifting property of bounded trees in dual spaces (this follows easily from a compactness argument, cf. [6]) there exists a tree $\left(z_{n}^{*}\right)$ in $B_{Y^{*}}$ such that $z_{n}^{*}(y)=y_{n}^{*}(y)$ for all $y$ in $Y_{0}$.

We claim that $\left(T^{*} z_{n}^{*}\right)$ is a Rademacher tree in $K$. To see this, first note that for each $k=1,2,3, \ldots$, letting $i=k+1$, the element $e_{i}$ of the usual $l_{1}$-basis satisfies $e_{n}^{*}\left(e_{i}\right)=(-1)^{n}$ for $n=2^{k}, \ldots, 2^{k+1}-1$. Taking each of the following sums from $n=2^{k}$ to $n=2^{k+1}-1$, we obtain

$$
\begin{aligned}
\left\|\sum(-1)^{n} T^{*} z_{n}^{*}\right\| & \geqslant\left|\sum(-1)^{n} T^{*} z_{n}^{*}\left(x_{i}\right)\right| \\
& =\|S\|^{-1}\left|\sum(-1)^{n} e_{n}^{*}\left(e_{i}\right)\right| \\
& =\|S\|^{-1}\left|\sum(-1)^{n}(-1)^{n}\right| \\
& =2^{k}\|S\|^{-1} .
\end{aligned}
$$

Hence $\left(T^{*} z_{n}^{*}\right)$ is a $\|S\|^{-1}$-Rademacher tree in $K$.

The second statement follows from the first statement and the observation following Theorem 1. This completes the proof.

A quick glance at the beginning of the above proof shows that any Banach space containing a bounded Rademacher tree fails to have the weak Radon-Nikodym property. Since the isomorphic copy of a Rademacher tree is again a Rademacher tree, this observation immediately shows that neither $c_{0}$ nor $L_{1}[0,1]$ can be embedded in a Banach space having the weak Radon-Nikodym property, a fact previously proved by Janicka [5] and by Ghoussoub and Saab [3].

III. Extreme points. In 1976, Haydon [4] showed that spaces not containing $l_{1}$ have fairly strong extremal properties in their duals. Since such duals also have the weak Radon-Nikodym property [5], the next theorem might be considered as a localization of Haydon's result. 
THEOREM 3. Each of the following statements about an operator $T: X \rightarrow Y$ implies all the others:

(a) The set $T^{*}\left(B_{Y^{*}}\right)$ is a weak Radon-Nikodym set.

(b) Every weak*-compact convex subset of $T^{*}\left(B_{Y^{*}}\right)$ is the norm-closed convex hull of its extreme points.

(c) For every weak ${ }^{*}$-compact subset $W$ of $T^{*}\left(B_{Y^{*}}\right)$, the weak ${ }^{*}$-closed convex hull of $W$ coincides with the norm-closed convex hull of $W$.

Consequently, a weak*-compact absolutely convex subset $K$ of $X^{*}$ is a weak Radon-Nikodym set if and only if every weak*-compact convex subset of $K$ is the norm-closed convex hull of its extreme points.

Proof. (a) $\Rightarrow$ (b). Let $K=T^{*}\left(B_{Y^{*}}\right)$ and let $C$ be a weak*-compact convex subset of $K$. Suppose $C \neq$ norm-cl conv(Ext $C$ ). By the proof of [4, Proposition 3.1], there exist a nonempty subset $S$ of $C$, a sequence $\left(x_{n}\right)$ in $B_{X}$, and a constant $\delta>0$ such that

$$
\delta \sum\left|a_{i}\right| \leqslant \sup \left\{\left|\sum a_{i} s\left(x_{i}\right)\right|: s \in S\right\}
$$

for all finitely nonzero sequences $\left(a_{i}\right)$ of reals. Fix $s$ in $S$ and observe that $s=T^{*} y^{*}$ for some $y^{*}$ in $B_{Y^{*}}$. Then

$$
\left|\sum a_{i} s\left(x_{i}\right)\right|=\left|\sum a_{i} T^{*} y^{*}\left(x_{i}\right)\right| \leqslant\left\|\sum a_{i}\left(T x_{i}\right)\right\| .
$$

Accordingly,

$$
\delta \sum\left|a_{i}\right| \leqslant\left\|\sum a_{i}\left(T x_{i}\right)\right\|,
$$

i.e., the sequence $\left(T x_{n}\right)$ is a copy of the $l_{1}$-basis. Consequently $T\left(B_{X}\right)$ is not weakly precompact. Appeal to Theorem 1 to see that $T^{*}\left(B_{Y^{*}}\right)$ is not a weak RadonNikodym set.

(b) $\Rightarrow$ (c). This follows immediately from the observations that if $W$ is a weak*compact subset of $K$, then weak*-cl conv $(W) \subseteq K$ and $\operatorname{Ext}\left(\right.$ weak $^{*}$-cl $\left.\operatorname{conv}(W)\right) \subseteq W$ $[1$, V.1.3].

(c) $\Rightarrow$ (a). Suppose $T^{*}\left(B_{Y^{*}}\right)$ is not a weak Radon-Nikodym set. Use Theorem 1 and Rosenthal's theorem to find a copy $\left(T x_{n}\right)$ of the usual $l_{1}$-basis in $T\left(B_{X}\right)$. Let $X_{0}$ denote the closed subspace determined by the sequence $\left(x_{n}\right)$ and let $i: X_{0} \rightarrow X$ be the natural inclusion map. In addition, let $T_{0}$ denote the restriction of $T$ to the subspace $X_{0}$. Note that since there is a $\delta>0$ satisfying

$$
\delta\left\|\sum a_{i} x_{i}\right\| \leqslant \delta \sum\left|a_{i}\right| \leqslant\left\|\sum a_{i} T x_{i}\right\|=\left\|T_{0}\left(\sum a_{i} x_{i}\right)\right\|
$$

for all finitely nonzero sequences $\left(a_{i}\right)$ of reals, the operator $T_{0}$ has a bounded inverse on its (closed) range $Y_{0}$. Therefore its adjoint $T_{0}^{*}: Y_{0}^{*} \rightarrow X_{0}^{*}$ also has a bounded inverse.

Let $V: Y_{0} \rightarrow C[0,1]$ be a quotient map on the separable space $Y_{0}$, and let $K_{0}$ denote the image of the set of unit point masses on $[0,1]$ under the action of the adjoint operator $V^{*}$. Then $K_{0}$ is a weak*-compact subset of $Y_{0}^{*}$ which has distinct weak*-closed and norm-closed convex hulls (see [4]). Since weak*-cl $\operatorname{conv}\left(K_{0}\right)$ is weak*-compact and $T_{0}^{*}$ is weak*-to-weak* continuous, we have

$$
T_{0}^{*}\left(\text { weak }^{*} \text {-cl } \operatorname{conv}\left(K_{0}\right)\right)=\text { weak }^{*} \text {-cl } \operatorname{conv}\left(T_{0}^{*} K_{0}\right) \text {. }
$$


In addition, since $T_{0}^{*}$ has a norm continuous inverse,

$$
T_{0}^{*}\left(\operatorname{norm}-\mathrm{cl} \operatorname{conv}\left(K_{0}\right)\right)=\text { norm-cl } \operatorname{conv}\left(T_{0}^{*} K_{0}\right) .
$$

Therefore norm-cl conv $\left(T_{0}^{*} K_{0}\right) \neq$ weak $^{*}$-cl $\operatorname{conv}\left(T_{0}^{*} K_{0}\right)$.

Let $j: Y_{0} \rightarrow Y$ denote the natural inclusion map. Pick a weak*-compact subset $M$ of $Y^{*}$ such that $j^{*} M=K_{0}$. Let $W=T^{*} M$ and observe that $i^{*} W=T_{0}^{*} K_{0}$. Then

$$
i^{*}\left(\text { weak }^{*} \text {-cl } \operatorname{conv}(W)\right)=\text { weak }^{*} \text {-cl } \operatorname{conv}\left(T_{0}^{*} K_{0}\right)
$$

and

$$
i^{*}(\operatorname{norm}-\mathrm{cl} \operatorname{conv}(W)) \subseteq \operatorname{norm}-\operatorname{cl} \operatorname{conv}\left(T_{0}^{*} K_{0}\right),
$$

so consequently weak*-cl $\operatorname{conv}(W) \neq \operatorname{norm}-\mathrm{cl} \operatorname{conv}(W)$. A suitable scalar multiple of $W$ produces a weak*-compact subset of $T^{*}\left(B_{Y^{*}}\right)$ with distinct weak*-closed and norm-closed convex hulls, as required.

This completes the proof.

IV. Dentability. In [11] Saab and Saab introduce the following dentability criterion.

Definition. A bounded subset $A$ of $X^{*}$ is said to be weak*-scalarly dentable if for every $\varepsilon>0$ and every $x^{* *}$ in $X^{* *}$ there exists a weak*-open slice $S=\left\{x^{*} \in A\right.$ : $\left.x^{*}(x)>\sup _{y^{*} \in A} y^{*}(x)-\alpha\right\}$ for some $x$ in $X$ and $\alpha>0$ such that the oscillation of $x^{* *}$ on $S$ satisfies

$$
\sup \left\{\left|x^{* *}\left(x^{*}\right)-x^{* *}\left(y^{*}\right)\right|: x^{*}, y^{*} \in S\right\} \leqslant \varepsilon .
$$

They then proceed to show that every nonempty bounded subset of $X^{*}$ is weak*-scalarly dentable if and only if for every weak*-compact subset $A$ and every $x^{* *}$ in $X^{* *}$, the restriction of $x^{* *}$ to $A$ equipped with the weak*-topology has a point of continuity. A close examination of their proof, though, reveals that it works inside any weak*-compact convex set; i.e. for a weak*-compact convex set $K$, every nonempty subset of $K$ is weak*-scalarly dentable if and only if for every weak*-compact subset $M$ of $K$ and every $x^{* *}$ in $X^{* *}$, the restriction of $x^{* *}$ to $(M$, weak*) has a point of continuity. In Riddle, Saab, and Uhl [8], however, this latter condition is shown to characterize the weak Radon-Nikodym property for $K$ provided that $K$ is absolutely convex.

With the help of a standard separation argument, it is easily seen that a nonempty bounded set $A$ is weak*-scalarly dentable if and only if for every $\varepsilon>0$ and every $x^{* *}$ in $X^{* *}$ there exists $x^{*}$ in $A$ such that

$$
x^{*} \notin \text { weak }^{*} \text {-cl } \operatorname{conv}\left(A \backslash\left\{y^{*} \in A:\left|x^{* *}\left(x^{*}\right)-x^{* *}\left(y^{*}\right)\right|<\varepsilon\right\}\right) .
$$

Observe that what is being removed from the set $A$ is a weak neighborhood of the point $x^{*}$.

It is perhaps worth remarking here what happens if various parts of this dentability condition are changed. If, for example, one takes the weak closure (or equivalently the norm closure), then the Bishop-Phelps theorem ensures that every nonempty subset of $X^{*}$ satisfies the new condition (as was pointed out to us by Elias $\mathrm{Saab}$ ). This immediately shows that weakly compact sets are weak*-scalarly dentable, a result that is not surprising since such sets are actually dentable. Likewise, if 
one requires the conditions to hold only for every $x$ in $X$ rather than all elements in $X^{* *}$, then again all nonempty subsets have the corresponding property. Finally, if one requires that the norm diameter of the weak*-open slice be less than $\varepsilon$, then the property is called weak*-dentability and every nonempty bounded set has the property if and only if $X^{*}$ has the Radon-Nikodym property (see Namioka and Phelps [7]).

The last theorem summarizes the discussion of this and the previous sections.

THEOREM 4. Each of the following statements about a weak*-compact absolutely convex subset $K$ of a conjugate Banach space $X^{*}$ implies all the others:

(a) The set $K$ is a weak Radon-Nikodym set.

(b) The set $K$ does not contain a Rademacher tree.

(c) Every weak*-compact convex subset of $K$ is the norm-closed convex hull of its extreme points.

(d) Every nonempty subset of $K$ is weak*-scalarly dentable.

ACKNOWLEDGEMENT. The author is grateful for the constant advice and support offered by Professor J. J. Uhl, Jr. during the preparation of the doctoral thesis from which this paper is taken.

\section{REFERENCES}

1. M. Day, Normed linear spaces, 3rd ed., Ergebnisse der Math. und ihrer Grenzgebiete, SpringerVerlag, Berlin and New York, 1973.

2. J. Diestel and J. J. Uhl, Jr., Vector measures, Math. Surveys, no. 15, Amer. Math. Soc., Providence, R. I., 1977.

3. N. Ghoussoub and E. Saab, On the weak Radon-Nikodym property, Proc. Amer. Math. Soc. 81 (1981), 81-84.

4. R. Haydon, Some more characterizations of Banach spaces containing $l_{1}$, Math. Proc. Cambridge Philos. Soc. 80 (1976), 269-276.

5. L. Janicka, Some measure-theoretical characterization of Banach spaces, not containing $l_{1}$, Bull. Acad. Polon. Sci. Sér. Sci. Math. 27 (1979), 561-565.

6. P. W. McCartney, Neighborly bushes and the Radon-Nikodym property for Banach spaces, Pacific J. Math. 87 (1980), 157-168.

7. I. Namioka and R. R. Phelps, Banach spaces which are Asplund spaces, Duke Math. J. 42 (1975), 735-750.

8. L. H. Riddle, E. Saab and J. J. Uhl, Jr., Sets with the weak Radon-Nikodym property in dual Banach spaces, Indiana Univ. Math. J. (to appear).

9. L. H. Riddle and J. J. Uhl, Jr., The fine line between Asplund spaces and spaces not containing a copy of $l_{1}$, preprint.

10. H. P. Rosenthal, A characterization of Banach spaces containing $l_{1}$, Proc. Nat. Acad. Sci. U.S.A. 71 (1974), 2411-2413.

11. E. Saab and P. Saab, On Banach spaces not containing $l_{1}$, Pacific J. Math. (to appear).

Department of Mathematics, Emory University, Atlanta, Georgia 30322 